\title{
Online Learning in Adversarial Lipschitz Environments
}

\author{
Odalric-Ambrym Maillard and Rémi Munos \\ SequeL Project, INRIA Lille - Nord Europe, France \\ \{odalric.maillard,remi.munos\}@inria.fr
}

\begin{abstract}
We consider the problem of online learning in an adversarial environment when the reward functions chosen by the adversary are assumed to be Lipschitz. This setting extends previous works on linear and convex online learning. We provide a class of algorithms with cumulative regret upper bounded by $\tilde{O}(\sqrt{d T \ln (\lambda)})$ where $d$ is the dimension of the search space, $T$ the time horizon, and $\lambda$ the Lipschitz constant. Efficient numerical implementations using particle methods are discussed. Applications include online supervised learning problems for both full and partial (bandit) information settings, for a large class of non-linear regressors/classifiers, such as neural networks.
\end{abstract}

\section{Introduction}

The adversarial online learning problem is defined as a repeated game between an agent (the learner) and an opponent, where at each round $t$, simultaneously the agent chooses an action (or decision, or arm, or state) $\theta_{t} \in \Theta$ (where $\Theta$ is a subset of $\mathbb{R}^{d}$ ) and the opponent chooses a reward function $f_{t}: \Theta \mapsto[0,1]$. The agent receives the reward $f_{t}\left(\theta_{t}\right)$. In this paper we will consider different assumptions about the amount of information received by the agent at each round. In the full information case, the full reward function $f_{t}$ is revealed to the agent after each round, whereas in the case of bandit information only the reward corresponding to its own choice $f_{t}\left(\theta_{t}\right)$ is provided.

The goal of the agent is to allocate its actions $\left(\theta_{t}\right)_{1 \leq t \leq T}$ in order to maximize the sum of obtained rewards $F_{T} \stackrel{\text { def }}{=} \sum_{t=1}^{T} f_{t}\left(\theta_{t}\right)$ up to time $T$ and its performance is assessed in terms of the best constant strategy $\theta \in \Theta$ on the same reward functions, i.e. $F_{T}(\theta) \stackrel{\text { def }}{=} \sum_{t=1}^{T} f_{t}(\theta)$. Defining the cumulative regret:

$$
R_{T}(\theta) \stackrel{\text { def }}{=} F_{T}(\theta)-F_{T},
$$

with respect to (w.r.t.) a strategy $\theta$, the agent aims at minimizing $R_{T}(\theta)$ for all $\theta \in \Theta$.

In this paper we consider the case when the functions $f_{t}$ are Lipschitz w.r.t. the decision variable $\theta$ (with Lipschitz constant upper bounded by $\lambda$ ). 
Previous results. Several works on adversarial online learning include the case of finite action spaces (the so-called learning from experts [1] and the multiarmed bandit problem [23]), countably infinite action spaces [4], and the case of continuous action spaces, where many works have considered strong assumptions on the reward functions, i.e. linearity or convexity.

In the online linear optimization (see e.g. 5667/8 in the adversarial case and 910 in the stochastic case) where the functions $f_{t}$ are linear, the resulting upper- and lower-bounds on the regret are of order (up to logarithmic factors) $\sqrt{d T}$ in the case of full information and $d^{3 / 2} \sqrt{T}$ in the case of bandit information [6] (and in good cases $d \sqrt{T}$ [5]). In online convex optimization $f_{t}$ is assumed to be convex 11 or $\sigma$-strongly convex 12, and the resulting upper bounds are of order $C \sqrt{T}$ and $C^{2} \sigma^{-1} \ln (T)$ (where $C$ is a bound on the gradient of the functions, which implicitly depends on the space dimension). Other extensions have been considered in 1314 15] and a minimax lower bound analysis in the full information case in [16. These results hold in bandit information settings where either the value or the gradient of the function is revealed.

To our knowledge, the weaker Lipschitz assumption that we consider here has not been studied in the adversarial optimization literature. However, in the stochastic bandit setting (where noisy evaluations of a fixed function are revealed), the Lipschitz assumption has been previously considered in [1718], see the discussion in Section 2.3.

Motivations: In many applications (such as the problem of matching ads to web-page contents on the Internet) it is important to be able to consider both large action spaces and general reward functions. The continuous space problem appears naturally in online learning, where a decision point is a classifier in a parametric space of dimension $d$. Since many non-linear non-convex classifiers/regressors have shown success (such as neural-networks, support vector machines, matching pursuits), we wish to extend the results of online learning to those non-linear non-convex cases. In this paper we consider a Lipschitz assumption (illustrated in the case of neural network architectures) which is much weaker than linearity or convexity.

What we do: We start in Section 1 by describing a general continuous version of the Exponentially Weighted Forecaster and state (Theorem 1) an upper bound on the cumulative regret of $O(\sqrt{d T \ln (d \lambda T)})$ under a non-trivial geometrical property of the action space. The algorithm requires, as a sub-routine, being able to sample actions according to continuous distributions, which may be impossible to do perfectly well in general.

To address the issue of sampling, we may use different sampling techniques, such as uniform grids, random or quasi-random grids, or use adaptive methods such as Monte-Carlo Markov chains (MCMC) or Population Monte-Carlo (PMC).

However, since any sampling technique introduces a sampling bias (compared to an ideal sampling from the continuous distribution), this also impacts the resulting performance of the method in terms of regret. This shows a tradeoff between 
regret and numerical complexity, which is illustrated by numerical experiments in Section 1.3 where PMC techniques are compared to sampling from uniform grids.

Then in Section 2 we describe several applications to learning problems. In the full information setting (when the desired outputs are revealed after each round), the case of regression is described in Section 2.1 and the case of classification in Section 2.2. Then Section 2.3 considers a classification problem in a bandit setting (i.e. when only the information of whether the prediction is correct or not is revealed). In the later case, we show that the expected number of mistakes does not exceed that of the best classifier by more than $O(\sqrt{d T K \ln (d \lambda T)})$, where $K$ is the number of labels. We detail a possible PMC implementation in this case.

We believe that the work reported in this paper provides arguments that the use of MCMC, PMC, and other adaptive sampling techniques is a promising direction for designing numerically efficient algorithms for online learning in adversarial Lipschitz environments.

\section{Adversarial Learning with Full Information}

We consider a search space $\Theta \subset \mathbb{R}^{d}$ equipped with the Lebesgue measure $\mu$. We write $\mu(\Theta)=\int_{\Theta} 1$. We assume that all reward functions $f_{t}$ have values in $[0,1]$ and are Lipschitz w.r.t. some norm $\|\cdot\|$ (e.g. $L_{1}, L_{2}$, or $L_{\infty}$ ) with a Lipschitz constant upper bounded by $\lambda>0$, i.e. for all $t \geq 1$ and $\theta_{1}, \theta_{2} \in \Theta$,

$$
\left|f_{t}\left(\theta_{1}\right)-f_{t}\left(\theta_{2}\right)\right| \leq \lambda\left\|\theta_{1}-\theta_{2}\right\| \text {. }
$$

\subsection{The ALF Algorithm}

We consider the natural extension of the EWF (Exponentially Weighted Forecaster) algorithm [19201] to the continuous action setting. Figure 1 describes this ALF algorithm (for Adversarial Lipschitz Full-information environment).

At each time step, the forecaster samples $\theta_{t}$ from a probability distribution $p_{t} \stackrel{\text { def }}{=} \frac{w_{t}}{\int_{\Theta} w_{t}}$ with $w_{t}$ being the weight function defined according to the previously observed reward functions $\left(f_{s}\right)_{s<t}$. The function $f_{t}$ is then revealed and the weight function is updated. We have $w_{t+1}(\theta)=\exp \left(\eta F_{t}(\theta)\right)$, and $\eta$ is a parameter of the algorithm.

Geometric considerations: The performance of the algorithm depends on the geometry of the space $\Theta \subset \mathbb{R}^{d}$ (relatively to the chosen norm), and since we want to derive bounds as a function of the dimension $d$, we now define classes of domains $\left(\left(\Theta_{d}\right)_{d \geq 0}\right.$ indexed by their dimension) with similar geometrical properties.

Definition 1. For the class of domains $\left(\Theta_{d}\right)_{d \geq 1}$, we define $\kappa(d)>1$ :

$$
\kappa(d) \stackrel{\text { def }}{=} \sup _{\theta \in \Theta_{d}, r>0} \frac{\min \left[\mu(B(\theta, r)), \mu\left(\Theta_{d}\right)\right]}{\mu\left(B(\theta, r) \cap \Theta_{d}\right)}
$$


Initialization: Set $w_{1}(\theta)=1$ for all $\theta \in \Theta$.

For each round $t=1,2, \ldots, T$

(1) Simultaneously the adversary chooses the reward function $f_{t}: \Theta \mapsto[0,1]$, and the learner chooses $\theta_{t} \stackrel{i i d}{\sim} p_{t}$, where $p_{t}(\theta) \stackrel{\text { def }}{=} \frac{w_{t}(\theta)}{\int_{\Theta} w_{t}(\theta) d \theta}$,

(2) The learner incurs the reward $f_{t}\left(\theta_{t}\right)$,

(3) The reward function $f_{t}$ is revealed to the learner. The weight function $w_{t}$ is updated as:

$$
w_{t+1}(\theta) \stackrel{\text { def }}{=} w_{t}(\theta) e^{\eta f_{t}(\theta)}, \text { for all } \theta \in \Theta
$$

Fig. 1. Adversarial Lipschitz learning algorithm in a Full-information setting (ALF algorithm)

Assumption $A 1$ There exists $\kappa>0$ such that $\kappa(d) \leq \kappa^{d}$, for all $d \geq 1$, and there exists $\kappa^{\prime}>0$ and $\alpha \geq 0$ such that $\mu(B(\theta, r)) \geq\left(r /\left(\kappa^{\prime} d^{\alpha}\right)\right)^{d}$ for all $r>0, d \geq 1$, and $\theta \in \mathbb{R}^{d}$.

The first part of this assumption says that $\kappa(d)$ scales at most exponentially with the dimension. This is reasonable if we consider domains with similar geometries (i.e. whenever the "angles" of the domains do not go to zero when the dimension $d$ increases). For example, in the domains $\Theta_{d}=[0,1]^{d}$, this assumption holds with $\kappa=2$ for any usual norm $\left(L_{1}, L_{2}\right.$ and $\left.L_{\infty}\right)$. The second part of the assumption about the volume of $d$-balls is a property of the norms and holds naturally for any usual norm: for example, $\kappa^{\prime}=1 / 2, \alpha=0$ for $L_{\infty}$, and $\kappa^{\prime}=\sqrt{\pi} /(\sqrt{2} e), \alpha=3 / 2$ for any norm $L_{p}, p \geq 1$, since for $L_{p}$ norms, $\mu(B(\theta, r)) \geq(2 r)^{d} / d$ ! and from Stirling formula, $d ! \sim \sqrt{2 \pi d}(d / e)^{d}$, thus $\mu(B(\theta, r)) \geq\left(r /\left(\frac{\sqrt{2 \pi}}{2 e} d^{3 / 2}\right)\right)^{d}$.

Remark 1. Notice that Assumption A1 makes explicit the required geometry of the domain in order to derive tight regret bounds.

We now provide upper-bounds for the ALF algorithm on the worst expected regret (i.e. $\sup _{\theta \in \Theta} \mathbb{E} R_{T}(\theta)$ ) and high probability bounds on the worst regret $\sup _{\theta \in \Theta} R_{T}(\theta)$.

Theorem 1. (ALF algorithm) Under Assumption A1, for any $\eta \leq 1$, the expected (w.r.t. the internal randomization of the algorithm) cumulative regret of the ALF algorithm is bounded as:

$$
\sup _{\theta \in \Theta} \mathbb{E} R_{T}(\theta) \leq T \eta+\frac{1}{\eta}\left[d \ln \left(c d^{\alpha} \eta \lambda T\right)+\ln (\mu(\Theta))\right],
$$

whenever $\left(d^{\alpha} \eta \lambda T\right)^{d} \mu(\Theta) \geq 1$, where $c \stackrel{\text { def }}{=} 2 \kappa \max \left(\kappa^{\prime}, 1\right)$ is a constant (which depends on the geometry of $\Theta$ and the considered norm). Under the same assumptions, with probability $1-\beta$, 


$$
\sup _{\theta \in \Theta} R_{T}(\theta) \leq T \eta+\frac{1}{\eta}\left[d \ln \left(c d^{\alpha} \eta \lambda T\right)+\ln (\mu(\Theta))\right]+\sqrt{2 T \ln \left(\beta^{-1}\right)} .
$$

We deduce that for the choice $\eta=\left(\frac{d}{T} \ln \left(c d^{\alpha} \lambda T\right)\right)^{1 / 2}$, when $\eta \leq 1$ and assuming $\mu(\Theta)=1$, we have:

$$
\sup _{\theta \in \Theta} \mathbb{E} R_{T}(\theta) \leq 2 \sqrt{d T \ln \left(c d^{\alpha} \lambda T\right)}
$$

and a similar bound holds in high probability.

The proof is given in Appendix A. Note that the parameter $\eta$ of the algorithm depends very mildly on the (unknown) Lipschitz constant $\lambda$. Actually even if $\lambda$ was totally unknown, the choice $\eta=\left(\frac{d}{T} \ln \left(c d^{\alpha} T\right)\right)^{1 / 2}$ would yield a bound $\sup _{\theta \in \Theta} \mathbb{E} R_{T}(\theta)=O(\sqrt{d T \ln (d T)} \ln \lambda)$ which is still logarithmic in $\lambda$ (instead of linear in the case of the discretization) and enables to consider classes of functions for which $\lambda$ may be large (and unknown).

Anytime algorithm. Like in the discrete version of EWF (see e.g. [21221]) this algorithms may easily be extended to an anytime algorithm (i.e. providing similar performance even when the time horizon $T$ is not known in advance) by considering a decreasing coefficient $\eta_{t}=\left(\frac{d}{2 t} \ln \left(c d^{\alpha} \lambda t\right)\right)^{1 / 2}$ in the definition of the weight function $w_{t}$. We refer to 22 for a description of the methodology.

The issue of sampling. In order to implement the ALF algorithm detailed in Figure 1 one should be able to sample $\theta_{t}$ from the continuous distribution $p_{t}$. However it is in general impossible to sample perfectly from arbitrary continuous distributions $p_{t}$, thus we need to resort to approximate sampling techniques, such as based on uniform grids, random or quasi-random grids, or adaptive methods such as Monte-Carlo Markov Chain (MCMC) methods or population MonteCarlo (PMC) methods. If we write $p_{t}^{N}$ the distribution from which the samples are actually generated, where $N$ stands for the computational resources (e.g. the number of grid points if we use a grid) used to generate the samples, then the expected regret $\mathbb{E} R_{T}(\theta)$ will suffer an additional term of at most $\sum_{t=1}^{T} \mid \int_{\Theta} p_{t} f_{t}-$ $\int_{\Theta} p_{t}^{N} f_{t} \mid$. This shows a tradeoff between the regret (low when $N$ is large, i.e. $p_{t}^{N}$ is close to $p_{t}$ ) and numerical complexity and memory requirement (which scales with $N$ ). In the next two sub-sections we discuss sampling techniques based on fixed grids and adaptive PMC methods, respectively.

\subsection{Uniform Grid over the Unit Hypercube}

A first approach consists in setting a uniform grid (say with $N$ grid points) before the learning starts and consider the naive approximation of $p_{t}$ by sampling at each round one point of the grid, since in that case the distribution has finite support and the sampling is easy.

Actually, in the case when the domain $\Theta$ is the unit hypercube $[0,1]^{d}$, we can easily do the analysis of an Exponentially Weighted Forecaster (EWF) playing on 
the grid and shows that the total expected regret is small provided that $N$ is large enough. Indeed, let $\Theta_{N} \stackrel{\text { def }}{=}\left\{\theta_{1}, \ldots, \theta_{N}\right\}$ be a uniform grid of resolution $h>0$, i.e. such that for any $\theta \in \Theta, \min _{1 \leq i \leq N}\left\|\theta-\theta_{i}\right\| \leq h$. This means that at each round $t$, we select the action $\theta_{I_{t}} \in \Theta_{N}$, where $I_{t} \stackrel{i i d}{\sim} p_{t}^{N}$ with $p_{t}^{N}$ the distribution on $\{1, \ldots, N\}$ defined by $p_{t}^{N}(i) \stackrel{\text { def }}{=} w_{t}(i) / \sum_{j=1}^{N} w_{t}(j)$, where the weights are defined as $w_{t}(i) \stackrel{\text { def }}{=} e^{\eta F_{t-1}\left(\theta_{i}\right)}$ for some appropriate constant $\eta=\sqrt{2 \ln N / T}$.

The usual analysis of EWF implies that the regret relatively to any point of the grid is upper bounded as: $\sup _{1 \leq i \leq N} \mathbb{E} R_{T}\left(\theta_{i}\right) \leq \sqrt{2 T \ln N}$.

Now, since we consider the unit hypercube $\Theta=[0,1]^{d}$, and under the assumption that the functions $f_{t}$ are $\lambda$-Lipschitz with respect to $L_{\infty}$-norm, we have that $F_{T}(\theta) \leq \min _{1 \leq i \leq N} F_{T}\left(\theta_{i}\right)+\lambda T h$. We deduce that the expected regret relatively to any $\theta \in \Theta$ is bounded as $\sup _{\theta \in \Theta} \mathbb{E} R_{T}(\theta) \leq \sqrt{2 T \ln N}+\lambda T h$.

Setting $N=h^{-d}$ with the optimal choice of $h$ in the previous bound (up to a logarithmic term) $h=\frac{1}{\lambda} \sqrt{d / T}$ gives the upper bound on the regret: $\sup _{\theta \in \Theta} \mathbb{E} R_{T}=O(\sqrt{d T \ln (\lambda \sqrt{T})})$.

However this discretized EWF algorithm suffers from severe limitations from a practical point of view:

1. The choice of the best resolution $h$ of the grid depends crucially on the knowledge of the Lipschitz constant $\lambda$ and has an important impact on the regret bound. However, usually $\lambda$ is not known exaclty (but an upper-bound may be available, e.g. in the case of neural networks discussed below). If we choose $h$ irrespective of $\lambda$ (e.g. $h=\sqrt{d / T})$ then the resulting bound on the regret will be of order $O(\lambda \sqrt{d T})$ which is much worst in terms of $\lambda$ than its optimal order $\sqrt{\ln \lambda}$.

2. The number of grid points (which determines the memory requirement and the numerical complexity of the EWF algorithm) scales exponentially with the dimension $d$.

Notice that instead of using a uniform grid, one may resort to the use of random (or quasi-random) grids with a given number of points $N$, which would scale better in high dimensions. However all those method are non-adaptive in the sense that the position of the grid point do not adapt to the actual reward functions $f_{t}$ observed through time. We would like to sample points according to an "adaptive discretization" that would allocate more points where the cumulative reward function $F_{t}$ is high. In the next sub-section we consider the ALF algorithm where we use adaptive sampling techniques such as MCMC and PMC which are designed for sampling from (possibly high dimensional) continuous distributions.

\subsection{A Population Monte-Carlo Sampling Technique}

The idea of sampling techniques such as Metropolis-Hasting ( $\mathrm{MH})$ or other MCMC (Monte-Carlo Markov Chain) methods (see e.g. 2324]) is to build a Markov chain that has $p_{t}$ as its equilibrium distribution, and starting from an 
initial distribution, iterates its transition kernel $K$ times so as to approximate $p_{t}$. Note that the rate of convergence of the distribution towards $p_{t}$ is exponential with $K$ (see e.g. [25]): $\delta(k) \leq(2 \epsilon)^{k / \tau(\epsilon)}$, where $\delta(k)$ is the total variation distance between $p_{t}$ and the distribution at step $k$, and $\tau(\epsilon)=\min \{k ; \delta(k) \leq \epsilon\}$ is the so called mixing time of the Markov Chain $(\epsilon<1 / 2)$.

Thus sampling $\theta_{t} \sim p_{t}$ only requires being able to compute $w_{t}(\theta)$ at a finite number of points $K$ (the number of transitions of the corresponding Markov chain needed to approximate the stationary distribution $p_{t}$ ). This is possible whenever the reward functions $f_{t}$ can be stored by using a finite amount of information, which is the case in the applications to learning, described in the next section.

However, using MCMC at each time step to sample from a distribution $p_{t}$ which is similar to the previous one $p_{t-1}$ (since the cumulative functions $F_{t}$ do not change much from one iteration to the next) is a waste of MC transitions. The exponential decay of $\delta(k)$ depends on the mixing time $\tau(\epsilon)$ which depends on both the target distribution and the transition kernel, and can be reduced when considering efficient methods based on interacting particles systems. The population Monte-Carlo (PMC) method (see e.g. [26]) approximates $p_{t}$ by a population of $N$ particles $\left(x_{t, k}^{1: N}\right)$ which evolve (during $1 \leq k \leq K$ rounds) according to a transition/selection scheme:

- At round $k$, the transition step generates a successor population $\widetilde{x}_{t, k}^{1: N} \stackrel{i i d}{\sim}$ $g_{t, k}\left(x_{t, k-1}^{1: N}, \cdot\right)$ according to a transition kernel $g_{t, k}(\cdot, \cdot)$. Then likelihood ratios are defined as $w_{t, k}^{1: N}=\frac{p_{t}\left(\widetilde{x}_{t, k}^{1: N}\right)}{g\left(x_{t, k-1}^{1: N}, \widetilde{x}_{t, k}^{1: N}\right)}$,

- The selection step resamples $N$ particles $x_{t, k}^{i}=\widetilde{x}_{t, k}^{I_{i}}$ for $1 \leq i \leq N$ where the selection indices $\left(I_{i}\right)_{1 \leq i \leq N}$ are drawn (with replacement) from the set $\{1 \ldots N\}$ according to a multinomial distribution with parameters $\left(w_{t, k}^{i}\right)_{1 \leq i \leq N}$

At round $K$, one particle (out of $N$ ) is selected uniformly randomly, which defines the sample $\theta_{t} \sim p_{t}^{N}$ that is returned by the sampling technique. Some properties of this approch is that the proposed sample tends to an unbiased independent sample of $p_{t}$ (when either $N$ or $K \rightarrow \infty$ ). We do not provide additional implementation details about this method here since this is not the goal of this paper, but we refer the interested reader to [26] for discussion about the choice of good kernels $g_{t, k}$ and automatic tuning methods of the parameter $K$ and number of particles $N$. Note that in [26], the authors prove a Central Limit Theorem showing that the term $\sqrt{N}\left(\int_{\Theta} p_{t} f-\int_{\Theta} p_{t}^{N} f\right)$ is asymptotically gaussian with explicit variance depending on the previous parameters (that we do not report here for it would require additional specific notations), thus giving the speed of convergence towards 0 . We also refer to [27] for known theoretical results of the general PMC theory.

When using this sampling techniques in the ALF algorithm, since the distribution $p_{t+1}$ does not differ much from $p_{t}$, we can initialize the particles at round $t+1$ with the particles obtained at the previous round $t$ at the last step of the 

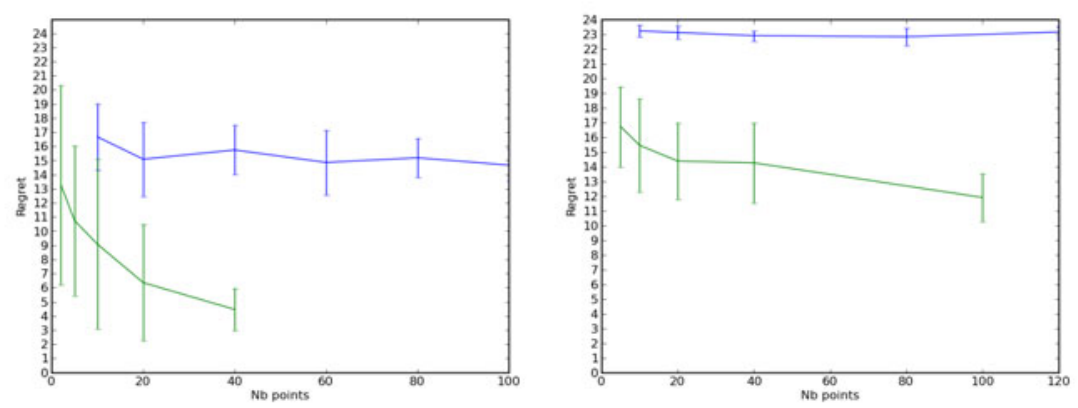

Fig. 2. Regret as a function of $N$, for dimensions $d=2$ (left figure) and 20 (right figure). In both figures, the top curve represents the grid sampling and the bottom curve the PMC sampling.

PMC sampling: $x_{t+1,1}^{i} \stackrel{\text { def }}{=} x_{t, K}^{i}$, for $1 \leq i \leq N$. In the numerical experiments reported in the next sub-section, this enabled to reduce drastically the number of rounds $K$ per time step (less than 5 in all experiments below).

\subsection{Numerical Experiments}

For illustation, let us consider the problem defined by: $\Theta=[0,1]^{d}, f_{t}(\theta)=(1-$ $\left.\left\|\theta-\theta_{t}\right\| / \sqrt{d}\right)^{3}$ where $\theta_{t}=t / T(1, \ldots, 1)^{\prime}$. The optimal $\theta^{*}\left(\right.$ i.e. $\left.\arg \max _{\theta} F_{T}(\theta)\right)$ is $1 / 2(1, \ldots, 1)^{\prime}$. Figure 2 plots the expected regret $\sup _{\theta \in \Theta} \mathbb{E} R_{T}(\theta)$ (with $T=100$, averaged over 10 experiments) as a function of the parameter $N$ (number of sampling points/particles) for two sampling methods: the random grid mentioned in the end of Section 1.2 and the PMC method. We considered two values of the space dimension: $d=2$ and $d=20$. Note that the uniform discretization technique is not applicable in the case of dimension $d=20$ (because of the curse of dimensionality). We used $K=5$ steps and used a Gaussian centered kernel $g_{t, k}$ of variance $\sigma^{2}=0.1$ for the PMC method.

Since the complexity of sampling from a PMC method with $N$ particles and from a grid of $N$ points is not the same, in order to compare the performance of the two methods both in terms of regret and runtime, we plot in Figure 3 the regret as a function of the CPU time required to do the sampling, for different values of $N$.

As expected, the PMC method is more efficient since its allocation of points (particles) depends on the cumulative rewards $F_{t}$ (it thus may be considered as an adaptive algorithm).

\section{Applications to Learning Problems}

\subsection{Online Regression}

Consider an online adversarial regression problem defined as follows: at each round $t$, an opponent selects a couple $\left(x_{t}, y_{t}\right)$ where $x_{t} \in \mathcal{X}$ and $y_{t} \in \mathcal{Y} \subset \mathbb{R}$, 

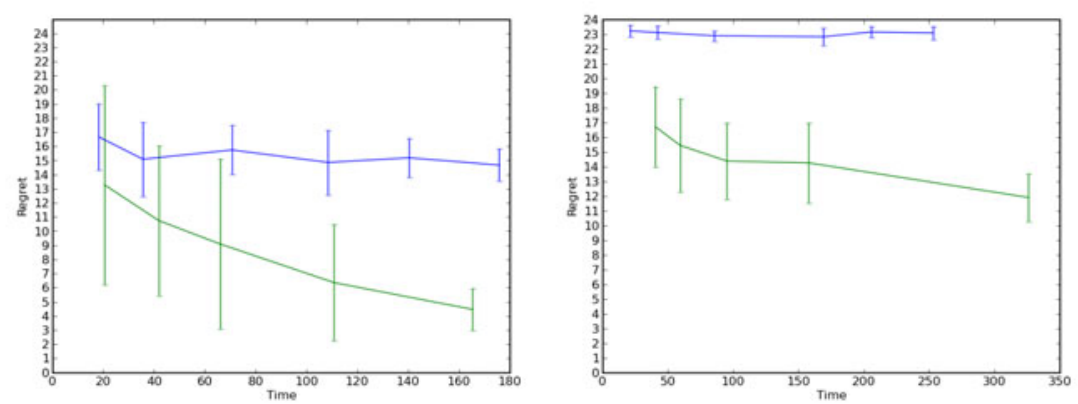

Fig. 3. Regret as a function of the CPU time used for sampling, for dimensions $d=2$ (left figure) and 20 (right figure). Again, in both figures, the top curve represents the grid sampling and the bottom curve the PMC sampling.

and shows the input $x_{t}$ to the learner. The learner selects a regression function $g_{t} \in \mathcal{G}$ and predicts $\hat{y}_{t}=g_{t}\left(x_{t}\right)$. Then the output $y_{t}$ is revealed and the learner incurs the reward (or equivalently a loss) $l\left(\hat{y}_{t}, y_{t}\right) \in[0,1]$.

Since the true output is revealed, it is possible to evaluate the reward of any $g \in \mathcal{G}$, which corresponds to the full information case.

Now, consider a parametric space $\mathcal{G}=\left\{g_{\theta}, \theta \in \Theta \subset \mathbb{R}^{d}\right\}$ of regression functions, and assume that the mapping $\theta \mapsto l\left(g_{\theta}(x), y\right)$ is Lipschitz w.r.t. $\theta$ with a uniform (over $x \in \mathcal{X}, y \in \mathcal{Y}$ ) Lipschitz constant $\lambda<\infty$. This happens for example when $\mathcal{X}$ and $\mathcal{Y}$ are compact domains, the regression $\theta \mapsto g_{\theta}$ is Lipschitz, and the loss function $(u, v) \mapsto l(u, v)$ is also Lipschitz w.r.t. its first variable (such as for e.g. $L_{1}$ or $L_{2}$ loss functions) on compact domains.

The online learning problem consists in selecting at each round $t$ a parameter $\theta_{t} \in \Theta$ such as to optimize the accuracy of the prediction of $y_{t}$ with $g_{\theta_{t}}\left(x_{t}\right)$. If we define $f_{t}(\theta) \stackrel{\text { def }}{=} l\left(g_{\theta}(x), y\right)$, then applying the ALF algorithm described previously (changing rewards into losses by using the transformation $u \mapsto 1-u$ ), we obtain directly that the expected cumulative loss of the ALF algorithm is almost as small as that of the best regression function in $\mathcal{G}$, in the sense that:

$$
\mathbb{E}\left[\sum_{t=1}^{T} l_{t}\right]-\inf _{g \in \mathcal{G}} \mathbb{E}\left[\sum_{t=1}^{T} l\left(g\left(x_{t}\right), y_{t}\right)\right] \leq 2 \sqrt{d T \ln \left(d^{\alpha} \lambda T\right)},
$$

where $l_{t} \stackrel{\text { def }}{=} l\left(g_{\theta_{t}}\left(x_{t}\right), y_{t}\right)$. To illustrate, consider a feedforward neural network (NN) 28] with parameter space $\Theta$ (the set of weights of the network) and one hidden layer. Let $n$ and $m$ be the number of input (respectively hidden) neurons. Thus if $x \in \mathcal{X} \subset \mathbb{R}^{n}$ is the input of the NN, a possible NN architecture would produce the output: $g_{\theta}(x) \stackrel{\text { def }}{=} \theta^{o} \cdot \sigma(x)$ with $\sigma(x) \in \mathbb{R}^{m}$ and $\sigma(x)_{l} \stackrel{\text { def }}{=} \sigma\left(\theta_{l}^{i} \cdot x\right)$ (where $\sigma$ is the sigmoid function) is the output of the $l$-th hidden neuron. Here $\theta=\left(\theta^{i}, \theta^{o}\right) \in \Theta \subset \mathbb{R}^{d}$ the set of (input, output) weights (thus here $\left.d=n \times m+m\right)$. 
The Lipschitz constant of the mapping $\theta \mapsto g_{\theta}(x)$ is upper bounded by $\sup _{x \in \mathcal{X}, \theta \in \Theta}\|x\|_{\infty}\|\theta\|_{\infty}$, thus assuming that the domains $\mathcal{X}, \mathcal{Y}$, and $\Theta$ are compacts, the assumption that $\theta \mapsto l\left(g_{\theta}(x), y\right)$ is uniformly (over $\mathcal{X}, \mathcal{Y}$ ) Lipschitz w.r.t. $\theta$ holds e.g. for $L_{1}$ or $L_{2}$ loss functions, and the previous result applies.

Now, as discussed above about the practical aspects of the ALF algorithm, in this online regression problem, the knowledge of the past input-output pairs $\left(x_{s}, y_{s}\right)_{s<t}$ enables to compute the weight $w_{t}(\theta)=\exp \left(\eta \sum_{s=1}^{t-1} l\left(g_{\theta}\left(x_{s}\right), y_{s}\right)\right)$ for any $\theta \in \Theta$, and thus enables to use a PMC algorithm to sample $\theta_{t}$ from the distribution $p_{t}$. Up to our knowledge, we believe this is a first regret bound analysis of online learning for non-linear NN regression, in an adversarial setting.

\subsection{Online Classification}

Now consider the problem of online classification (i.e. when the set of labels $\mathcal{Y}$ is finite). Here we can no longer make the assumption that the classifier's prediction $g_{\theta}(x) \in \mathcal{Y}$ is Lipschitz w.r.t. the parameter $\theta$ (and neither that the loss function $l\left(y, y^{\prime}\right)=\mathbb{I}_{\left\{y=y^{\prime}\right\}}$ is Lipschitz w.r.t. its first variable). One way to circumvent this problem is to consider a class $\mathcal{G}=\left\{g_{\theta}, \theta \in \Theta\right\}$ of stochastic classifiers, so that $g_{\theta}(y \mid x)$ represents the probability of predicting label $y$ given input $x$. The ALF algorithm would apply as follows: at round $t$, the algorithms chooses $\theta_{t} \in \Theta$ and samples the prediction $\hat{y}_{t}$ from the distribution $g_{\theta_{t}}\left(\cdot \mid x_{t}\right)$.

When the label $y_{t}$ is revealed, the loss function $f_{t}(\theta) \stackrel{\text { def }}{=} g_{\theta}\left(y_{t} \mid x_{t}\right)$ for all classifiers $g_{\theta}$ may be computed. Thus assuming that the mapping $\theta \mapsto g_{\theta}(y \mid x)$ is Lipschitz w.r.t. $\theta$ with uniform (over $\mathcal{X} \times \mathcal{Y}$ ) Lipschitz constant $\lambda$, then Theorem 1 applies, and we have that

$$
\underbrace{\sup _{g \in \mathcal{G}}\left\{\sum_{t=1}^{T} g\left(y_{t} \mid x_{t}\right)\right\}}_{\begin{array}{c}
\text { Exp. nb. of correct } \\
\text { predictions of best classifier }
\end{array}}-\underbrace{\mathbb{E}\left\{\sum_{t=1}^{T} g_{\theta_{t}}\left(y_{t} \mid x_{t}\right)\right\}}_{\begin{array}{c}
\text { Exp. nb. of correct } \\
\text { predictions of ALF algo. }
\end{array}} \leq 2 \sqrt{d T \ln \left(c d^{\alpha} \lambda T\right)}
$$

which says that the expected number of good predictions of the ALF algorithm is almost as good as that of the best classifier in $\mathcal{G}$. An example of such parametric regression setting is the case of neural networks (parameterized by $\theta$ ) where the activation of the output neurons (one for each label $y$ of $\mathcal{Y}$ ), up to some renormalization, define the probability distribution $g_{\theta}(y \mid x)$.

\subsection{Online Classification with Bandit Information}

In the previous section, the information revealed by the opponent enables to compute the reward (or loss) function $f_{t}(\theta)$ for all $\theta \in \Theta$. In the bandit information case considered now only the reward $f_{t}\left(\theta_{t}\right)$ of the selected action is revealed. Under our Lipschitz assumption on the functions, the knowledge of $f_{t}$ at a point $\theta_{t}$ reveals very few information about $f_{t}$ elsewhere. Thus we cannot expect to derive tight regret bounds in general. However we can obtain interesting bounds in the case when the reward function $f_{t}$ may actually be coded by a 
Initialization: Set $w_{1}(\theta)=1$ for all $\theta \in \Theta$.

For each round $t=1,2, \ldots, T$

(1) The adversary chooses $\left(x_{t}, y_{t}\right) \in \mathcal{X} \times \mathcal{Y}$ and shows $x_{t}$ to the learner,

(2) The learner chooses $\theta_{t} \sim p_{t}$, where $p_{t}(\theta) \stackrel{\text { def }}{=} \frac{w_{t}(\theta)}{\int_{\Theta} w_{t}(\theta) d \theta}$, and predicts $\hat{y}_{t} \sim$ $q_{t, \theta_{t}}$, where $q_{t, \theta}(y) \stackrel{\text { def }}{=}(1-\gamma) g_{\theta_{t}}\left(y \mid x_{t}\right)+\frac{\gamma}{K}$,

(3) The learner sees the (bandit) information $Z_{t} \stackrel{\text { def }}{=} \mathbb{I}_{\hat{y}_{t}=y_{t}}$, from which he defines $\tilde{f}_{t}(\theta) \stackrel{\text { def }}{=} \frac{g_{\theta}\left(\hat{y}_{t} \mid x_{t}\right)}{q_{t}\left(\hat{y}_{t}\right)} Z_{t}$, where $q_{t}(y) \stackrel{\text { def }}{=} \int_{\Theta} p_{t}(\theta) q_{t, \theta}(y) d \theta$, for any $y \in \mathcal{Y}$.

(4) The weight function $w_{t}$ is updated according to $w_{t+1}(\theta)=$ $w_{t}(\theta) e^{\eta \tilde{f}_{t}(\theta)}$, for all $\theta \in \Theta$.

Fig. 4. The Adversarial Lipschitz Bandit Classifier (ALBC algo)

finite amount of information. We illustrate this setting on the online classification problem described in Section 2.2 but with the difference that the true label $y_{t} \in \mathcal{Y}=\{1, \ldots, K\}$ is not revealed at each round: the only available information is $Z_{t} \stackrel{\text { def }}{=} \mathbb{I}_{\left\{\hat{y}_{t}=y_{t}\right\}}$, i.e. whether the prediction $\hat{y}_{t}$ is correct or not. An example of applications is the problem of web advertisement systems, where the user's click is the only received feedback.

Again, we consider a parametric family of stochastic classifiers $\mathcal{G}=\left\{g_{\theta}, \theta \in\right.$ $\Theta\}$, where $g_{\theta}(y \mid x)$ corresponds to the probability of selecting $y \in \mathcal{Y}$ given the input $x$. Now, in each round, a classifier $g_{\theta_{t}}$ is selected (by sampling $\theta_{t} \sim p_{t}$ ) and a prediction $\hat{y}_{t}$ is made. However, in this bandit setting, the feedback information $Z_{t}=\mathbb{I}_{\left\{\hat{y}_{t}=y_{t}\right\}}$ does not enable to evaluate the performance $f_{t}(\theta) \stackrel{\text { def }}{=} g_{\theta}\left(y_{t} \mid x_{t}\right)$ of any classifiers $g_{\theta}, \theta \in \Theta$. Instead, we randomize the prediction by considering a mixture distribution between $g_{\theta_{t}}$ and the uniform distribution: $\hat{y}_{t} \sim q_{t, \theta_{t}}$, where $q_{t, \theta}$ is the distribution over the labels $\mathcal{Y}$ defined by $q_{t, \theta}(y) \stackrel{\text { def }}{=}(1-\gamma) g_{\theta}\left(y \mid x_{t}\right)+\frac{\gamma}{K}$.

This idea is close to the Exp4 algorithm in [3]. Given the information $Z_{t}$, we build an estimate $\tilde{f}_{t}(\theta)$ of the performance $f_{t}(\theta)$ of any classifiers $g_{\theta}: \tilde{f}_{t}(\theta) \stackrel{\text { def }}{=}$ $\frac{g_{\theta}\left(\hat{y}_{t} \mid x_{t}\right)}{q_{t}\left(\hat{y}_{t}\right)} Z_{t}$, where $q_{t}(y) \stackrel{\text { def }}{=} \mathbb{E}_{\theta \sim p_{t}}\left[q_{t, \theta}(y)\right]$, for any $y \in \mathcal{Y}$. This estimate is unbiased since:

$$
\begin{aligned}
\mathbb{E}_{\theta_{t}, \hat{y}_{t}} \tilde{f}_{t}(\theta) & =\int_{\Theta} p_{t}\left(\theta^{\prime}\right) \sum_{y \in \mathcal{Y}} \frac{q_{t, \theta^{\prime}}(y) g_{\theta}\left(y \mid x_{t}\right)}{q_{t}(y)} \mathbb{I}_{\left\{y=y_{t}\right\}} d \theta^{\prime} \\
& =\int_{\Theta} p_{t}\left(\theta^{\prime}\right) \frac{q_{t, \theta^{\prime}}(y) g_{\theta}\left(y_{t} \mid x_{t}\right)}{q_{t}\left(y_{t}\right)} d \theta^{\prime}=g_{\theta}\left(y_{t} \mid x_{t}\right)=f_{t}(\theta)
\end{aligned}
$$

Figure 4 describes this Adversarial Lipschitz Bandit Classifier (ALBC) algorithm. The next result assesses the expected performance of the ALBC algorithm $\sum_{t=1}^{T} \mathbb{I}_{\left\{\hat{y}_{t}=y_{t}\right\}}$ in comparison with the expected performance of the best 
classifier $g \in \mathcal{G}$, in terms of number of correct predictions. Define the regret:

$$
R_{T}(\theta) \stackrel{\text { def }}{=} \sum_{t=1}^{T} g_{\theta}\left(y_{t} \mid x_{t}\right)-\mathbb{E}\left[\sum_{t=1}^{T} \mathbb{I}_{\left\{\hat{y}_{t}=y_{t}\right\}}\right] .
$$

The ALBC algorithm has a regret $\sup _{\theta \in \Theta} \mathbb{E} R_{T}(\theta) \leq 4 \sqrt{K d T \ln \left(c d^{\alpha} \lambda T\right)}$ (the proof is omitted from this extended abstract but follows the same lines as the proof of ALF algorithm combined with EXP4 ideas). Notice that like in the multi-armed bandit problem, in this bandit setting, the regret suffers from an additional factor $K$ per round (i.e. $\sqrt{T}$ is replaced by $\sqrt{K T}$ in the bound), compared to the full information case.

A practical algorithm. A practical implementation of the ALBC algorithm requires being able to sample $\theta_{t}$ from $p_{t}$. The key difference with the technique detailed in Section 1.3 is that in the ALBC algorithm, the functions $\tilde{f}_{t}(\theta)$ depend on $q_{t}\left(\hat{y}_{t}\right)$ which is not directly known. However a refined MCMC or PMC algorithm is possible: at round $t$, assume that we have kept in memory the information: $H_{<t} \stackrel{\text { def }}{=}\left\{\left(x_{s}, \hat{y}_{s}, Z_{s}, q_{s}\left(\hat{y}_{s}\right)\right)_{s<t}\right\}$.

We now show that (1) this is possible, and (2) this is sufficient for sampling $\theta_{t} \sim p_{t}$. We prove (1) recursively by showing that from $H_{<t}$ we are able to calculate $q_{t}\left(\hat{y}_{t}\right)$ (the other pieces of information $x_{t}, \hat{y}_{t}$, and $Z_{t}$ are available at the end of round $t$ ). Thus we only need to prove that from $H_{<t}$, we can sample $\theta_{t} \sim p_{t}$ and compute $q_{t}\left(\hat{y}_{t}\right)$. But since $q_{t}\left(\hat{y}_{t}\right)$ is the expectation of $q_{t, \theta}\left(\hat{y}_{t}\right)$ for $\theta \sim p_{t}$, we may consider a MCMC or PMC method where the same Markov chain (having $p_{t}$ as stationnary distribution) or particle population serves for both sampling $\theta_{t} \sim p_{t}$ and estimating $q_{t}\left(\hat{y}_{t}\right)$. Finally, this is possible since the pointwise evaluation of $w_{t}$ (thus of $p_{t}$ up to a renormalization constant) only depends on information in $H_{<t}$.

\section{Conclusion}

We have considered the adversarial online learning framework in the case of Lipschitz functions. In the full information case, the bound shows the same rate $\sqrt{d T}$ as for linear functions. This enables to derive similar performance bounds for online regression and classification, thus extending previous results to non-linear parametric approximation, such as neural networks. Our main contribution was to consider a continuous extension of the EWF algorithm (ALF algorithm) for which we provide geometrical conditions for sound regret analysis, and discuss the use of different approximation schemes and especially the use of a PMC sampling method compared to non adaptive sampling methods. We provided experiments showing the benefit of using a PMC sampling method for minimizing regret under computational time constraint compared to naive random grid.

We applied this result to derive bounds for (full information) regression and classification online learning problems and (bandit information) $K$-classes classification problems where the revealed information is the correctness of the 
prediction. We derived a regret bound on the expected number of mistakes of order $\sqrt{d T K}$, and illustrate the case of a Neural Networks architecture.

\section{Acknowledgment}

This work has been supported by French National Research Agency (ANR) through COSINUS program (project EXPLO-RA number ANR-08-COSI-004).

\section{References}

1. Cesa-Bianchi, N., Lugosi, G.: Prediction, Learning, and Games. Cambridge University Press, New York (2006)

2. Auer, P., Cesa-bianchi, N., Freund, Y., Schapire, R.E.: Gambling in a rigged casino: The adversarial multi-armed bandit problem. In: Proceedings of the 36th Annual Symposium on Foundations of Computer Science, pp. 322-331. IEEE Computer Society Press, Los Alamitos (1995)

3. Auer, P., Cesa-bianchi, N., Freund, Y., Schapire, R.E.: The non-stochastic multiarmed bandit problem. SIAM Journal on Computing 32 (2002)

4. Poland, J.: Nonstochastic bandits: Countable decision set, unbounded costs and reactive environments. Theor. Comput. Sci. 397(1-3), 77-93 (2008)

5. Dani, V., Hayes, T., Kakade, S.: The price of bandit information for online optimization. In: Platt, J., Koller, D., Singer, Y., Roweis, S. (eds.) Advances in Neural Information Processing Systems 20, pp. 345-352. MIT Press, Cambridge (2008)

6. Abernethy, J., Hazan, E., Rakhlin, A.: Competing in the dark: An efficient algorithm for bandit linear optimization. In: Servedio, R.A., Zhang, T. (eds.) Conference on Learning Theory, pp. 263-274. Omnipress (2008)

7. Cesa-Bianchi, N., Lugosi, G.: Combinatorial bandits. In: Conference on Learning Theory (2009)

8. Kakade, S.M., Shalev-Shwartz, S., Tewari, A.: Efficient bandit algorithms for online multiclass prediction. In: Proceedings of the 25th International Conference on Machine learning, pp. 440-447. ACM, New York (2008)

9. Auer, P.: Using confidence bounds for exploitation-exploration trade-offs. Journal of Machine Learning Research, 397-422 (2002)

10. Dani, V., Hayes, T.P., Kakade, S.M.: Stochastic linear optimization under bandit feedback (2008) (in submission)

11. Zinkevich, M.: Online convex programming and generalized infinitesimal gradient ascent. In: International Conference on Machine learning, pp. 928-936 (2003)

12. Hazan, E., Agarwal, A., Kale, S.: Logarithmic regret algorithms for online convex optimization. In: Conference on Learning Theory, pp. 499-513 (2006)

13. Bartlett, P., Hazan, E., Rakhlin, A.: Adaptive online gradient descent. In: Platt, J.C., Koller, D., Singer, Y., Roweis, S.T. (eds.) Advances in Neural Information Processing Systems. MIT Press, Cambridge (2007)

14. Shalev-Shwartz, S.: Online Learning: Theory, Algorithms, and Applications. PhD thesis (July 2007)

15. Flaxman, A.D., Kalai, A.T., McMahan, H.B.: Online convex optimization in the bandit setting: gradient descent without a gradient. In: Proceedings of the sixteenth annual ACM-SIAM Symposium on Discrete algorithms, pp. 385-394. SIAM, Philadelphia (2005) 
16. Abernethy, J.D., Bartlett, P., Rakhlin, A., Tewari, A.: Optimal strategies and minimax lower bounds for online convex games. Technical Report UCB/EECS-2008-19, EECS Department, University of California, Berkeley (February 2008)

17. Kleinberg, R., Slivkins, A., Upfal, E.: Multi-armed bandit problems in metric spaces. In: Proceedings of the 40th ACM Symposium on Theory of Computing, pp. 681-690 (2008)

18. Bubeck, S., Munos, R., Stoltz, G., Szepesvári, C.: Online optimization of X-armed bandits. In: Advances in Neural Information Processing Systems (2008)

19. Littlestone, N., Warmuth, M.: The weighted majority algorithm. Information and Computation 108, 212-261 (1994)

20. Cesa-Bianchi, N., Freund, Y., Haussler, D., Helmbold, D.P., Shapire, R., Warmuth, M.: How to use expert advice. Journal of the ACM 44(3), 427-485 (1997)

21. Auer, P., Cesa-bianchi, N., Gentile, C.: Adaptive and self-confident on-line learning algorithms. Journal of Computer and System Sciences 64 (2000)

22. Stoltz, G.: Incomplete information and internal regret in prediction of individual sequences. PhD thesis (2005)

23. Gilks, W., Richardson, S., Spiegelhalter, D.: Markov Chain Monte Carlo in Practice. Chapman Hall/CRC, Boca Raton (1996)

24. Andrieu, C., De Freitas, N., Doucet, A., Jordan, M.: An introduction to mcmc for machine learning. Journal of Machine Learning Research 50, 5-43 (2003)

25. Levin, D.A., Peres, Y., Wilmer, E.L.: Markov Chains and Mixing Times. American Mathematical Society, Providence (2008)

26. Douc, R., Guillin, A., Marin, J., Robert, C.: Minimum variance importance sampling via population monte carlo. Esaim P\&S 11 (2007)

27. Del Moral, P.: Feynman-Kac formulae: genealogical and interacting particle systems with applications/Pierre Del Moral, p. 555. Springer, Heidelberg (2004)

28. Bishop, C.M.: Pattern Recognition and Machine Learning (Information Science and Statistics). Springer, Heidelberg (2006)

29. Devroye, L., Györfi, L., Lugosi, G.: A Probabilistic Theory of Pattern Recognition. Springer, New York (1996)

\section{A Proof of Theorem 1 (ALF algorithm)}

We start by following the usual proof for exponentially weighted forecasting. Define $W_{t} \stackrel{\text { def }}{=} \int_{\Theta} w_{t}$. For any $t \in\{1, \ldots, T\}$, we have:

$$
\frac{W_{t+1}}{W_{t}}=\frac{\int_{\Theta} \exp \left(\eta F_{t}\right)}{\int_{\Theta} \exp \left(\eta F_{t-1}\right)}=\int_{\Theta} p_{t}(\theta) \exp \left(\eta f_{t}(\theta)\right) \text {. }
$$

Since $\exp (u) \leq 1+u+u^{2}$ for $u \leq 1$, then, whenever $\eta \leq 1$, we have $\frac{W_{t+1}}{W_{t}} \leq$ $1+\eta \int_{\Theta} p_{t} f_{t}+\eta^{2} \int_{\Theta} p_{t} f_{t}^{2}$. Moreover, since $W_{1}=\mu(\Theta)$, we get:

$$
\ln \left(W_{T+1}\right) \leq \eta \sum_{t=1}^{T} \int_{\Theta} p_{t} f_{t}+T \eta^{2}+\ln (\mu(\Theta)) .
$$

Let us write $h(\theta) \stackrel{\text { def }}{=} \exp \left(\eta F_{T}(\theta)\right)$, and $h^{*} \stackrel{\text { def }}{=} \max _{x \in \Theta} h(\theta)$. We have that

$$
\begin{aligned}
\left|h\left(\theta_{1}\right)-h\left(\theta_{2}\right)\right| & \leq \eta\left|F_{T}\left(\theta_{1}\right)-F_{T}\left(\theta_{2}\right)\right| h^{*} \\
& \leq \eta \lambda T h^{*}|| \theta_{1}-\theta_{2}||,
\end{aligned}
$$


since the function $F_{T}$ is $\lambda T$-Lipschitz. Let $\theta^{*}$ be any point of maximum of $h$, and define $\pi(\theta) \stackrel{\text { def }}{=} \max \left(0,1-\eta \lambda T\left\|\theta-\theta^{*}\right\|\right)$. Then for all $\theta \in \Theta$,

$$
h(\theta) \geq h^{*} \pi(\theta) .
$$

Indeed, this holds for any $\theta \notin B\left(\theta^{*}, 1 /(\eta \lambda T)\right)$ where $B(\theta, r)$ is the ball $\left\{x^{\prime}, \| x-\right.$ $\left.x^{\prime} \| \leq r\right\}$, since in that case, $\pi(\theta)=0$. Now if there were some $\theta \in B\left(\theta^{*}, 1 /(\eta \lambda T)\right)$ such that $h(\theta)<h^{*} \pi(\theta)$, then we would have: $h\left(\theta^{*}\right)-h(\theta)>\eta \lambda T h^{*}\left\|x-x^{*}\right\|$, which would contradict the Lipschitz property (5) of $h$.

Notice that $\pi$ is a pyramid function with base $B\left(\theta^{*}, 1 /(\eta \lambda T)\right)$ and height 1 . We now state a Lemma that will enable us to derive a lower bound on $\int_{\Theta} \pi$.

Lemma 1. For any $\theta^{*} \in \Theta, r>0$, let $\pi$ be the function defined by $\pi(\theta) \stackrel{\text { def }}{=}$ $\max \left(0,1-\left\|x-x^{*}\right\| / r\right)$. Then:

$$
\int_{\Theta} \pi \geq \frac{1}{(d+1) \kappa(d)} \min \left[\mu\left(B\left(\theta^{*}, r\right)\right), \mu(\Theta)\right]
$$

Proof.

$$
\begin{aligned}
\int_{\Theta} \pi & =\int_{\mathbb{R}^{D}} \mathbb{I}_{\theta \in \Theta \cap B\left(\theta^{*}, r\right)}\left(1-\frac{\left\|\theta^{*}-\theta\right\|}{r}\right) \mu(d \theta) \\
& =\int_{\mathbb{R}^{D}} \mathbb{I}_{\theta \in \Theta \cap B\left(\theta^{*}, r\right)} \int_{0}^{1} \mathbb{I}_{\left\|\theta^{*}-\theta\right\| \leq \alpha r} d \alpha \mu(d \theta) \\
& =\int_{0}^{1} \int_{\mathbb{R}^{D}} \mathbb{I}_{\theta \in \Theta \cap B\left(\theta^{*}, \alpha r\right)} \mu(d \theta) d \alpha \\
& =\int_{0}^{1} \mu\left(\Theta \cap B\left(\theta^{*}, \alpha r\right)\right) d \alpha
\end{aligned}
$$

Now, using the definition of $\kappa(d)$ from (11),

$$
\int_{\Theta} \pi \geq \int_{0}^{1} \frac{1}{\kappa(d)} \min \left[\alpha^{d} \mu\left(B\left(\theta^{*}, r\right)\right), \mu(\Theta)\right] d \alpha
$$

We deduce that if $\mu(\Theta) \geq \mu\left(B\left(\theta^{*}, r\right)\right)$ then $\int_{\Theta} \pi \geq \frac{\mu\left(B\left(\theta^{*}, r\right)\right)}{(d+1) \kappa(d)}$. And otherwise, $\exists \alpha_{0}<1$ such that $\mu(\Theta)=\alpha_{0}^{d} \mu\left(B\left(\theta^{*}, r\right)\right)$ thus we have $\int_{\Theta} \pi \geq \frac{\mu(\Theta)}{\kappa(d)}\left(1-\alpha_{0}+\right.$ $\left.\frac{\alpha_{0}}{d+1}\right) \geq \frac{\mu(\Theta)}{(d+1) \kappa(d)}$ and the Lemma is proved.

We apply this Lemma with the $\pi$ function and $r=1 / \eta \lambda T$ to obtain:

$$
\int_{\Theta} \pi \geq \frac{1}{(d+1) \kappa(d)} \min \left[\mu\left(B\left(\theta^{*}, \frac{1}{\eta \lambda T}\right)\right), \mu(\Theta)\right]
$$

Now using (6) together with the previous bound combined with Assumption A1 (i.e. $\kappa(d) \leq \kappa^{d}$ and $\mu\left(B\left(\theta^{*}, r\right)\right) \geq\left(r /\left(\kappa^{\prime} d^{\alpha}\right)^{d}\right)$, we derive the lower bound:

$$
\int_{\Theta} h \geq h^{*} \min \left[\frac{1}{\left(c d^{\alpha} \eta \lambda T\right)^{d}}, \frac{\mu(\Theta)}{c^{d}}\right] .
$$

where we set $c=2 \kappa \max \left(\kappa^{\prime}, 1\right)$. 
From its definition, $W_{T+1}=\int_{\Theta} h$, thus

$$
\ln \left(W_{T+1}\right) \geq \eta \max _{\theta \in \Theta} F_{T}(\theta)-\ln \left(\max \left[\left(c d^{\alpha} \eta \lambda T\right)^{d}, \frac{c^{d}}{\mu(\Theta)}\right]\right),
$$

which, together with (4) yields:

$$
\sup _{\theta \in \Theta} F_{T}(\theta)-\sum_{t=1}^{T} \int_{\Theta} p_{t} f_{t} \leq T \eta+\frac{1}{\eta} \max \left[d \ln \left(c d^{\alpha} \eta \lambda T\right)+\ln (\mu(\Theta)), d \ln c\right] .
$$

Since $\int_{\Theta} p_{t} f_{t}=\mathbb{E}_{t}\left[f_{t}\left(\theta_{t}\right)\right]$, where $\mathbb{E}_{t}$ denotes the expectation w.r.t. the choice of $\theta_{t} \sim p_{t}$, we deduce that the expected regret (w.r.t. the internal randomization of the learner) of any $\theta \in \Theta$ is bounded according to:

$$
\mathbb{E} R_{T}(\theta) \leq T \eta+\frac{1}{\eta}\left(d \ln \left(c d^{\alpha} \eta \lambda T\right)+\ln (\mu(\Theta))\right)
$$

whenever $d \ln \left(d^{\alpha} \eta \lambda T\right) \geq-\ln (\mu(\Theta))$.

Now, for the high probability result, if we introduce $Y_{t}=\int_{\Theta} p_{t} f_{t}-f_{t}\left(\theta_{t}\right)$ and $\mathcal{F}_{<t}$ the $\sigma$-algebra generated by the past random decisions, then $\mathbb{E}\left[Y_{t} \mid \mathcal{F}_{<t}\right]=0$, thus $Y_{1}, \ldots, Y_{T}$ is a martingale difference sequence, and since $f_{t} \in[0,1],\left|Y_{t}\right| \leq$ 1 a.s., using Hoeffding-Azuma's inequality (see e.g. [29]), we obtain that with probability at least $1-\beta$,

$$
\sum_{t=1}^{T} \int_{\Theta} p_{t} f_{t} \leq F_{T}+\sqrt{2 T \ln \left(\beta^{-1}\right)}
$$

which enables to deduce (3). 